\title{
Elastic properties of surfactant monolayers at liquid-liquid interfaces: A molecular dynamics study
}

\section{Laradji, Mohamed; Mouritsen, Ole G.}

Published in:

Journal of Chemical Physics

Link to article, DOI:

$10.1063 / 1.481486$

Publication date:

2000

Document Version

Publisher's PDF, also known as Version of record

Link back to DTU Orbit

Citation (APA):

Laradji, M., \& Mouritsen, O. G. (2000). Elastic properties of surfactant monolayers at liquid-liquid interfaces: A molecular dynamics study. Journal of Chemical Physics, 112(19), 8621-8630. https://doi.org/10.1063/1.481486

\section{General rights}

Copyright and moral rights for the publications made accessible in the public portal are retained by the authors and/or other copyright owners and it is a condition of accessing publications that users recognise and abide by the legal requirements associated with these rights.

- Users may download and print one copy of any publication from the public portal for the purpose of private study or research.

- You may not further distribute the material or use it for any profit-making activity or commercial gain

- You may freely distribute the URL identifying the publication in the public portal

If you believe that this document breaches copyright please contact us providing details, and we will remove access to the work immediately and investigate your claim 


\title{
Elastic properties of surfactant monolayers at liquid-liquid interfaces: A molecular dynamics study
}

\author{
Mohamed Laradjia) \\ Department of Physics, University of Prince Edward Island, Charlottetown, Prince Edward Island, \\ C1A 4P3, Canada \\ Ole G. Mouritsen \\ Department of Chemistry, Technical University of Denmark, DK-2800 Lyngby, Denmark
}

(Received 22 November 1999; accepted 9 February 2000)

\begin{abstract}
Using a simple molecular model based on the Lennard-Jones potential, we systematically study the elastic properties of liquid-liquid interfaces containing surfactant molecules by means of extensive and large-scale molecular dynamics simulations. The main elastic constants of the interface, corresponding to the interfacial tension and the mean bending modulus are determined from the analyses of the long-wavelength behavior of the structure factor of the capillary waves. We found that the interfacial tension decreases with increasing surfactant interfacial coverage and/or surfactant chain length. However, we found that the corresponding change in the bending rigidity is nonmonotonic. Specifically, we found that the bending rigidity decreases with increasing surfactant interfacial coverage for small surfactant interface coverages, but then it increases as the surfactant interface coverage is further increased. Using a Gaussian theory on an interfacial Ginzburg-Landau model of surfactants, we find that the initial decrease of the bending rigidity is attributed to coupling between fluctuations of the surfactant orientation field to those in the interfacial height. (C) 2000 American Institute of Physics. [S0021-9606(00)70717-3]
\end{abstract}

\section{INTRODUCTION}

Ternary mixtures of two immiscible fluids and surfactant molecules can form stable isotropic microemulsions characterized by mesoscopically segregated water and oil domains, separated by adsorbed surfactant monolayers. ${ }^{1,2}$ Microemulsions are found in ternary mixtures of water, oil and amphiphilic surfactants, or in ternary mixtures of two homopolymers and a diblock copolymer. Microemulsions form when the entropy of mixing of the incompatible fluids is balanced by the reduction of interfacial energy as a result of the adsorption of the surfactant molecules. The phase behavior of ternary mixtures containing surfactant molecules is for the most part dictated by the elastic properties of the surfactant monolayers. There has consequently been a great interest in attempting to extract the elastic constants of surfactant monolayers experimentally as well as theoretically. The study of the elastic properties of surfactant monolayers is also useful for systems of biological relevance such as lipid bilayers. In general, the leading contributions to the energy of an interface of area $\mathcal{A}$ can be expressed in the Helfrich form, ${ }^{3}$ in terms of its local principal curvatures $c_{1}(\mathbf{r})$ and $c_{2}(\mathbf{r})$ :

$$
\mathcal{H}=\int^{\mathcal{A}} d a\left[\sigma+\frac{\kappa}{2}\left(c_{1}+c_{2}-2 c_{0}\right)^{2}+\bar{\kappa} c_{1} c_{2}+\cdots\right],
$$

where the constants $\sigma, \kappa$ and $\bar{\kappa}$ are the interfacial tension, mean bending rigidity modulus, and Gaussian or saddlesplay bending rigidity modulus, respectively. $c_{0}$ is a charac-

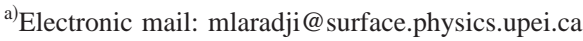

teristic spontaneous (or preferred) curvature. In the case of a bare interface or an interface with a small interface coverage of surfactants, $\kappa$ and $\bar{\kappa}$ are very small, and the interfacial energy is dominated by the interfacial tension. However, when the interface coverage of surfactants is large, such as in the case of a microemulsion internal interface, the interfacial tension becomes vanishingly small, and the bending terms dominate the interfacial energy. Typical experimental estimates of the bending moduli in the microemulsion phase are $\kappa \sim k_{B} T$ and $\bar{\kappa} \approx-2 \kappa .^{4-6}$

There have been a large amount of theoretical studies investigating the phase behavior of ternary mixtures of two immiscible fluids and surfactants. These range from the phenomenological coarse-grained theories to microscopic, mostly lattice, theories. ${ }^{1,2}$ Coarse-grained phenomenological models consider the ternary mixture as two mesoscopically segregated regions, separated by surfactant monolayers with specific elastic coefficients. These models have been successful in predicting the correct bulk phase behavior of these systems. Other coarse-grained models are based on Ginzburg-Landau free-energy functionals of one or more order parameters. These models have also been successful in predicting a correct phase behavior, and they have furthermore been useful for inferring structural properties of the interface. ${ }^{7}$ Unfortunately, it is not clear how to relate the microscopic properties of the surfactant molecules to the various empirical constants in coarse-grained models.

Microscopic theories were also developed to determine the effect of short rigid surfactants ${ }^{8}$ or long flexible surfactants $^{9,10}$ on the interfacial tension of two immiscible fluids. Unfortunately, most of these calculations were based 
on mean-field approximations. Few molecular dynamics studies have been performed to extract the interfacial elastic constants for relatively short surfactants. ${ }^{11}$ In a very recent molecular dynamics simulation on self-assembled surfactant bilayers, Goetz et al. ${ }^{12}$ extracted the elastic coefficients of a tensionless membrane (surfactant bilayer) from the analysis of its capillary waves.

The recent development of the self-consistent field theory for flexible polymers has contributed to an important progress in our understanding of the phase behavior of polymeric systems. Hong and Noolandi ${ }^{13}$ extended this theory to the study of diblock copolymers at homopolymerhomopolymer interfaces, and analyzed the effect of diblock copolymers on the interfacial tension of the interface. Wang and Safran ${ }^{14}$ analyzed the effect of diblock copolymers on the bending properties of an interface in the limit of strongly stretched diblock copolymer chains. Later, Matsen and Schick $^{15}$ studied these effects using a single order-parameter Ginzburg-Landau theory in conjunction with Leibler's theory for diblock copolymer melts. ${ }^{16}$ Recently, Laradji and Desai ${ }^{17}$ extended their self-consistent theory of anisotropic composition fluctuations in diblock copolymer melts ${ }^{18}$ to obtain results on the effect of diblock copolymer monolayers on the interfacial tension and bending rigidity of a homopolymer-homopolymer interface through the analyses of the long-wavelength capillary waves. Similar results were recently obtained by Matsen ${ }^{19}$ using the self-consistent field theory alone on fixed spherical and cylindrical geometries. Also recently, Müller and Schick ${ }^{20}$ and Werner et al. ${ }^{21}$ performed Monte Carlo simulations of ternary mixtures of two immiscible homopolymers and diblock copolymers using the bond-fluctuation model, and inferred as well as the effect of the copolymers on the interfacial tension and bending rigidity of the interface.

In the present article, we investigate the effect of surfactants on the elastic properties of fluid-fluid interfaces using large-scale molecular dynamics simulations, via the analysis of the long-wavelength fluctuations of the interface. The advantages of using the molecular dynamics method lie in the fact that within this approach, realistic models for surfactant molecules can be used, and that the method allows for an average over a wide portion of the phase space, making this method more "exact" than the often used mean-field theories. We found that the interfacial tension decreases as the surfactant interfacial coverage or the surfactant chain length is increased. However, we found that the bending rigidity coefficient may exhibit a nonmonotonic behavior. Namely for small surfactant interface coverages, the bending rigidity decreases as the surfactant interface coverage is increased, but then it eventually increases with a further increase of the surfactant interface coverage. These results are in excellent qualitative agreement with the recent calculation based on a theory of anisotropic fluctuations by Laradji and Desai. ${ }^{17}$

The paper is organized as follows: The details of the molecular model and the simulational techniques, regarding the molecular dynamics method used by us and the method for tracing the interface, are presented in Sec. II. In Sec. III, the results of our simulations are presented. We summarize and onclude in Sec. IV. In the Appendix, we describe a fieldtheoretical model for an interface containing surfactants.

\section{MODEL AND SIMULATION TECHNIQUES}

\section{A. Interaction potential}

We first describe the molecular model which will be used in our simulations. Let us consider a three-dimensional system composed of $N_{\mathrm{A}}$ A mono-atomic molecules, $N_{\mathrm{B}}$ B mono-atomic molecules, and $N_{s}$ poly-atomic surfactants composed of a flexible linear sequence of $l_{s} / 2$ A-particles attached to another linear sequence of $l_{s} / 2 \mathrm{~B}$-particles via anharmonic springs. The system is therefore composed of only two types of "atoms" with their total number being $N=N_{\mathrm{A}}+N_{\mathrm{B}}+l_{\mathrm{s}} N_{\mathrm{s}}$. The particles interact via a generalized Lennard-Jones potential, inspired by the Weeks-ChandlerAndersen work, ${ }^{22}$

$$
\begin{aligned}
U_{\alpha_{i} \alpha_{j}}\left(\mathbf{r}_{i j}\right)= & 4 \epsilon\left\{\left(\frac{\delta}{r_{i j}}\right)^{12}-\left(\frac{\delta}{r_{i j}}\right)^{6}\right]-\left[\left(\frac{\delta}{r_{\alpha_{i} \alpha_{j}}^{c}}\right)^{12}\right. \\
& \left.\left.-\left(\frac{\delta}{r_{\alpha_{i} \alpha_{j}}^{c}}\right)^{6}\right]\right\} \theta\left(r_{\alpha_{i} \alpha_{j}}^{c}-r_{i j}\right),
\end{aligned}
$$

where $\theta(x)$ is the usual Heaviside function and

$$
\alpha_{i}=\left\{\begin{array}{l}
1, \quad \text { if } i \text { is an A particle, } \\
-1, \quad \text { if } i \text { is a B particle. }
\end{array}\right.
$$

In the potential above, the A and B "atoms" within a surfactant molecule are also represented by $\alpha_{i}$. In Eq. (2), $r_{i j}$ is the distance separating the $i$ th from the $j$ th molecule, and $r_{\alpha_{i} \alpha_{j}}^{c}$ is a cutoff distance which depends on the species of the interacting pair, and is chosen such that the potential is the usual cut-and-shifted Lennard-Jones potential for particles belonging to the same species, but is purely repulsive for particles belonging to different species, i.e.,

$$
r_{\alpha_{i} \alpha_{j}}^{c}= \begin{cases}2.5 \delta, & \text { if } \alpha_{i}=\alpha_{j}, \\ 2^{1 / 6} \delta, & \text { if } \alpha_{i} \neq \alpha_{j} .\end{cases}
$$

In the absence of surfactants, the phase diagram of the model, which has recently been calculated numerically by Toxvaerd and Velasco $^{23}$ via a semi-grand-canonical ensemble Monte Carlo simulation, exhibits a two-phase region at low temperatures ending in a consolute point at $T_{c}=(4.7$ $\pm 0.2) \epsilon / k_{\mathrm{B}}$ for a fluid number density of $\rho=0.8 \delta^{-3}$. The connectivity between the particles composing a surfactant molecule is ensured through a symmetric generalized Lennard-Jones potential defined by

$$
U_{\alpha_{i} \alpha_{j}}^{s}\left(r_{i j}\right)=\left\{\begin{array}{l}
U_{\alpha_{i} \alpha_{j}}\left(r_{i j}\right)+\frac{\epsilon}{2}\left|\alpha_{i}+\alpha_{j}\right|, \quad \text { if } r_{i j} \leqslant 2^{1 / 6} \delta, \\
U_{\alpha_{i} \alpha_{j}}\left(2^{7 / 6} \delta-r_{i j}\right)+\frac{\epsilon}{2}\left|\alpha_{i}+\alpha_{j}\right|, \\
\text { if } 2^{1 / 6} \delta<r_{i j}<2^{7 / 6} \delta .
\end{array}\right.
$$

The Hamiltonian of the system can therefore be written 


$$
\begin{aligned}
\mathcal{H}\left(\left\{\mathbf{p}_{i}\right\},\left\{\mathbf{r}_{i}\right\}\right)= & \sum_{i=1}^{N} \frac{\mathbf{p}_{i}^{2}}{2 m}+\frac{1}{2} \sum_{i \neq j}^{N} U_{\alpha_{i} \alpha_{j}}\left(\mathbf{r}_{i}-\mathbf{r}_{j}\right) \\
& +\sum_{p=1}^{N_{\mathrm{s}}} \sum_{i=p}^{p\left(l_{\mathrm{s}}-1\right)} U_{\alpha_{i} \alpha_{i+1}}^{s}\left(\mathbf{r}_{i}-\mathbf{r}_{i+1}\right),
\end{aligned}
$$

where $m$ is the mass of one particle (here we assume that all "atoms" have the same mass), and $\mathbf{p}_{i}$ is the momentum of "atom" $i$.

\section{B. Molecular dynamics method}

All particles are placed in a three-dimensional box of size $L_{x y}^{2} \times L_{z}$. An interface between the A-rich and B-rich phases, parallel to the $x y$-plane is forced into the system by using anti-periodic boundary conditions in the z-direction: When a particle crosses the boundary along the $z$-direction, it switches to the other species, and an A-particle near for example the upper boundary interacts with the B-particles across the lower boundary as if they belong to the same species, but interacts with the A-particles across the same boundary as if they were B-particles. Along the $x$ - and $y$-directions, usual periodic boundary conditions were used. Our choice for these boundary conditions is different from the commonly used periodic boundary conditions in all directions. The latter conditions will force the existence of at least two interfaces in the system. Since we are limited in general by a relatively small number of particles in the system, the average distance between the two interfaces will be small, leading to interface-interface interactions that are likely to invalidate the results. We should emphasize, however, that our choice of these boundary conditions cannot be used for studying the vapor-liquid interfacial properties of one-component systems, for example.

In our molecular dynamics simulations, the temperature is controlled by a Nosé-Hoover thermostat, ${ }^{24-26}$ in which the particles move according to the following Hamilton equations:

$$
\begin{aligned}
& \frac{d}{d t} \mathbf{r}_{i}(t)=\frac{\mathbf{p}_{i}(t)}{m}, \\
& \frac{d}{d t} \mathbf{p}_{i}(t)=\mathbf{f}_{i}(t)-\eta(t) \mathbf{p}_{i}(t),
\end{aligned}
$$

where $\mathbf{f}_{i}(t)=\nabla_{i} \mathcal{H}$, is the force acting on particle $i$ due to all other particles within the range of interaction, Eq. (4), and $\eta(t)$ is the thermostat time-dependent friction parameter which varies with the total excess kinetic energy according to

$$
\frac{d}{d t} \eta(t)=\left(\sum_{i=1}^{N} \frac{\mathbf{p}_{i}^{2}}{m_{i}}-g k_{\mathrm{B}} T\right) /\left(g k_{\mathrm{B}} T \tau_{\eta}^{2}\right),
$$

where $g=3(N-1)$ is the total number of degrees of freedom in the system, and $\tau_{\eta}$ is a characteristic thermostat relaxation time. The effect of $\eta$ is such that the molecules gain or lose speed if the average kinetic energy per degree of freedom is lower or higher than $k_{\mathrm{B}} T / 2$, respectively.

The Hamilton equations (7) are integrated using the leap-frog algorithm,

$$
\begin{aligned}
& \mathbf{r}_{i}(t+\delta t)=\mathbf{r}_{i}(t)+\frac{\delta t}{m} \mathbf{p}_{i}(t)\left(t+\frac{\delta t}{2}\right) \\
& \mathbf{p}_{i}\left(t+\frac{\delta t}{2}\right)=\frac{\delta t}{1+\frac{\delta t}{2} \eta(t)} \mathbf{f}_{i}(t)+\frac{1-\frac{\delta t}{2} \eta(t)}{1+\frac{\delta t}{2} \eta(t)} \mathbf{p}_{i}\left(t-\frac{\delta t}{2}\right)
\end{aligned}
$$

In our simulation, we have specifically considered $N$ $=50,000$ particles with a fluid number density $\rho=0.8 \delta^{-3}$ in a box with an aspect ratio $L_{z} / L_{x y}=0.4$. The time step was chosen to be $\delta t=0.005 \tau$ and the friction characteristic time $\tau_{\eta}=0.03 \tau$, where the time scale is $\tau=\sqrt{m \sigma^{2} / \epsilon}$. All simulations were performed at a temperature $k_{\mathrm{B}} T=\epsilon$. Our choice of a relatively low temperature is required in order to have a well defined interface which is traceable, while at the same time this temperature is well above the solid-gas transition temperature. Many surfactant interface coverages (defined by $\left.c_{s}=N_{s} / L_{x y}^{2}\right)$ and two surfactant chain lengths, corresponding to $l_{s}=4$ and 8 , were considered in this study.

\section{Tracing the interface}

Due to the A-B symmetry in our model, the interface does not prefer to bend towards the A- or the B-fluid. Therefore, the spontaneous curvature $c_{0}$ vanishes in our case. If we suppose that the interfacial fluctuations are gentle, then the Hamiltonian in Eq. (1) can be rewritten in terms of a singlevalued function $h(\mathbf{x})$, where $\mathbf{x}=(x, y)$, describing the distance of the interface from its mean position,

$$
\mathcal{H}(\{h\})=\int d \mathbf{x}\left[\frac{\sigma}{2}\left(\nabla_{\mathbf{x}} h\right)^{2}+\frac{\kappa}{2}\left(\nabla_{\mathbf{x}}^{2} h\right)^{2}+\cdots\right] .
$$

The structure of the interface can be inferred from the structure factor, defined as the Fourier transform of the heightheight correlation function,

$$
S(\mathbf{q})=\frac{1}{L^{2}}\left\langle\left|\widetilde{h}_{\mathbf{q}}\right|^{2}\right\rangle=\left\langle\left|\int^{L} d \mathbf{x} e^{-i \mathbf{q} \cdot \mathbf{x}} h(\mathbf{x})\right|^{2}\right\rangle,
$$

where $\mathbf{q}=\left(q_{x}, q_{y}\right)$ is the lateral wave-vector, and $\tilde{h}_{\mathbf{q}}$ is the Fourier component of $h(x, y)$. In Eq. (10), the term involving the Gaussian bending rigidity has been omitted since it is a topological constant, and therefore its contribution cannot be inferred from the analysis of the fluctuations of the interface. If the higher order powers $h$ are neglected in Eq. (10), and the equipartition theorem is invoked, one finds that the interfacial height-height structure factor is given by

$$
S(\mathbf{q})=\frac{2 k_{B} T}{\sigma q^{2}+\kappa q^{4}+\mathcal{O}\left(q^{6}\right)} .
$$

We trace the interface through the following algorithm: "Ghost", particles, or plaquettes, are introduced in the system at the interface and are allowed to move at every time step. A ghost particle while feeling the neighboring A- and B-particles in the system, does not affect their dynamics, and consequently does not affect the physics of the system. The system is divided into $64 \times 64$ square grids along the $x y$-plane, and each grid point is occupied by a plaquette. 
This ensures that the interface position along the $z$-axis remains a single-valued function. Each plaquette is allowed to move only along the $z$-axis according to the following rules.

(1) In the beginning of the simulation, a flat interface, at $h_{0}$, separates the A-rich at the lower part of the system from the B-rich phase, at the upper part of the system. All plaquettes are at height $h_{0}$.

(2) For each plaquette, at a point $(x, y)$, we assign two values, $z_{1}$ and $z_{2}$ to the actual position of the plaquette, i.e., $z_{1}=h_{0}$ and $z_{2}=h_{0}$.

(3) We search for all particles belonging to the A-species which are above the plaquette, and have $(x, y)$ positions within the area of the plaquette. $z_{1}$ is then assigned the $z$-coordinate of the A-particle which is closest above the plaquette position. Likewise, we then search for all B-species particles which are below the plaquette, and have positions $(x, y)$ within the area of the plaquette. $z_{2}$ is then assigned the $z$-coordinate of the B-particle closest below the plaquette.

(4) At time step $t+\delta t / 2$, we assign a new position of the plaquette $h(x, y)=\left(z_{1}+z_{2}\right) / 2$.

We found that this algorithm gives a very accurate position of the interface for relatively low temperatures at which the A- and B-particles are very well segregated.

\section{RESULTS AND DISCUSSION}

\section{A. Real space configurations and concentration profiles}

In Figs. 1 and 2, typical cuts of our systems along directions normal to the $x y$-plane are shown for systems with surfactant lengths $l_{s}=4$ and 8 , respectively, and for various interface coverages. In the case of $l_{s}=4$, it is clear that the interfacial roughness is increased as the surfactant interface coverage is increased. In particular, for the case of high interface coverages, as in the case of systems $\left(l_{s}=4, N_{s}\right.$ $=1400)$ or $\left(l_{s}=8, N_{s}=1250\right)$, the interface looks like an elastic sheet with fluctuations which are suppressed at intermediate wavelengths, an indication that as the surfactant interface coverage is increased, the interfacial fluctuations becomes more dominated by the bending rigidity. We should point out that for the system $\left(l_{s}=8, N_{s}=1250\right)$, we found that the width of the interface eventually becomes comparable to the system size in the $z$-direction, indicating that the interface might be unstable for this set of parameters. Indeed, as we will see later in our discussion of the interfacial tension, the interfacial tension for systems with $l_{s}=8$ vanishes at about $N_{s}=1100$ (see Fig. 7).

The structure of the interface can be inferred in more detail through the number density profiles along the $z$-axis, defined as

$$
\phi_{\alpha}(z)=\frac{1}{L^{2}}\left\langle\int d \mathbf{x} \phi_{\alpha}(\mathbf{x}, z)\right\rangle,
$$

for the A- and B-particles, and

$$
\phi_{\alpha}^{s}(z)=\frac{1}{L^{2}}\left\langle\int d \mathbf{x} \phi_{\alpha}^{s}(\mathbf{x}, z)\right\rangle,
$$

for the $\alpha$-part of the surfactants.
The density profiles along the $z$-axis for a variety of various surfactant coverages are shown in Figs. 3 and 4 for surfactant lengths $l_{s}=4$ and $l_{s}=8$, respectively. In all cases, these profiles clearly show that the A- and B-particles are very well segregated, and that almost all surfactants are adsorbed at the interfaces. These figures also show that the total fluid density is slightly lower than the average density in the interfacial region, as a result of the repulsive interactions between the A- and B-particles. However, as the amount of surfactants is increased, the amount of depletion is decreased, signaling the compatibilization effect of the surfactants. For systems containing longer surfactants (Fig. 4), we notice small damped oscillations in the fluid density around the interface. These oscillations were not observed in the previous simulations done on ternary mixtures of two immiscible homopolymers and diblock copolymers. ${ }^{21,27}$ Figures 3 and 4 also show that the A-monomers (B-monomers) of surfactants are mainly located in the A-rich (B-rich) region of the system. However a small mixed region is observed at the interface. The main source for this mixed region is not a local mixing of the A- and B-monomers of the surfactants, but rather due to the fluctuations of the interface and the fact that density profiles are being averaged over the $x y$-planes. Figures 3 and 4 show that as the surfactant interface coverage is increased; the amount of surfactant buildup at the interfaces is also increased. However, in the case of the shorter surfactants with high interface coverages [e.g., Fig. 3(d)], we found that the density of surfactants in the interfacial area is reduced while the region where the surfactants are found is widened: This is not due to a local decrease of the surfactant density, but rather to the fact that densities have been averaged over the $x y$-planes.

\section{B. Structure factor and elastic constants}

In Fig. 5, the interfacial structure factor versus the wavevector $q$ is shown, on a logarithmic scale, for the binary system, and for a ternary system containing $N_{s}=1000$ surfactants of length $l_{s}=8$. In the binary case, this figure clearly shows that for small wavevectors, $S(q) \propto q^{-2}$, as expected from capillary-wave theory. This behavior indicates that the long-wavelength fluctuations of the interface are controlled by the interfacial tension. For the system with $N_{s}=1000$ and $l_{s}=8$, the deviation of $\ln S(q)$ from the $q^{-2}$ behavior is clear, indicating that for high surfactant interface coverages, the intermediate wavelengths interfacial fluctuations become also influenced by the bending rigidity.

The elastic constants of the interface can be extracted from examining the structure factor at small wavevectors. The interfacial tension, $\sigma$ is obtained from the intercept of $1 / q^{2} S(q)$, vs $q^{2}$, with the $y$-axis. The mean bending rigidity corresponds to the slope of $1 / q^{2} S(q)$, as illustrated in Fig. 6 . Before presenting our results for the elastic coefficients, it is worthwhile to discuss the structure factor as plotted in Fig. 6. This figure clearly shows the deviation of the structure factor from Eq. (11). It is interesting to note that an almost identical form of the structure factor was also observed in the calculations of Laradji and Desai ${ }^{17}$ on ternary mixtures of two homopolymers and diblock copolymers. 

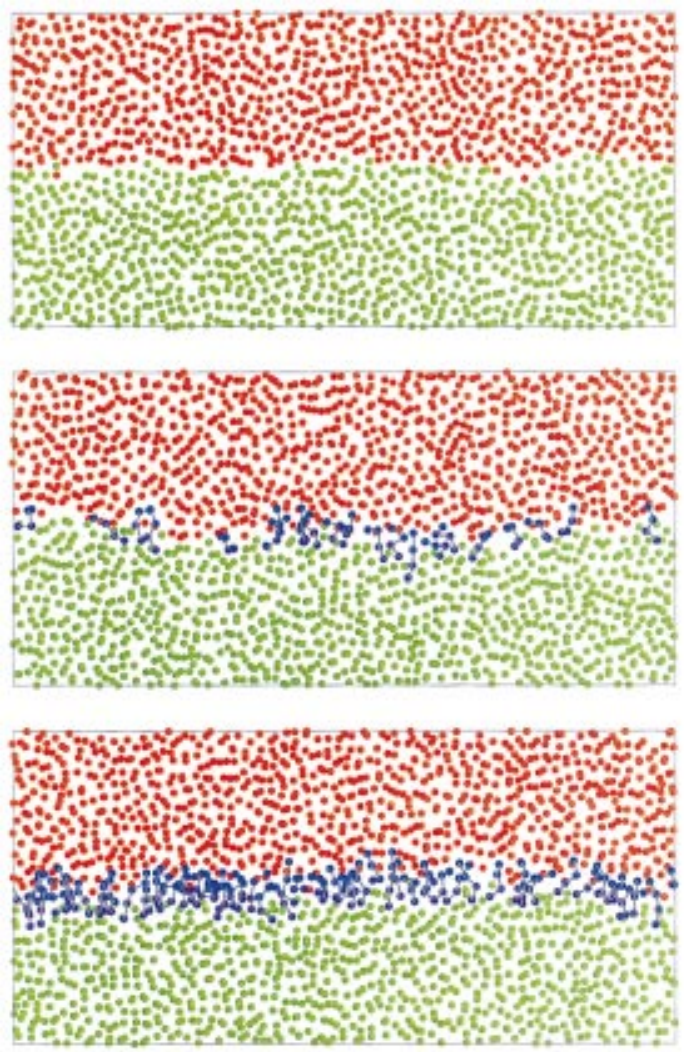

(c)
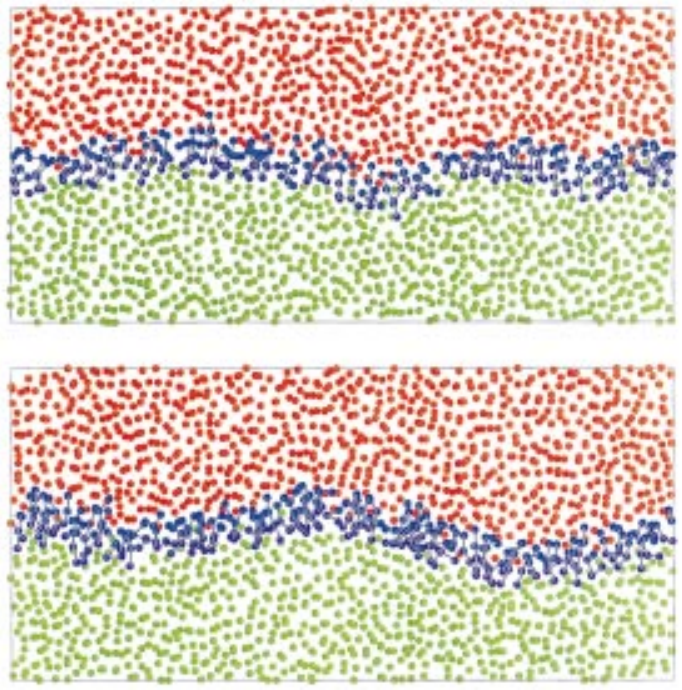

(e)

FIG. 1. (Color) Typical cuts of the three-dimensional configurations along the $x z$-plane for the case of surfactants with length $l_{s}=4$. Interface coverages shown correspond to (a) $N_{s}=0$, (b) $N_{s}=400$, (c) $N_{s}=1000$, (d) $N_{s}$ $=1250$, and (e) $N_{s}=1400$.

The deviation of the structure factor in the simulation from that derived from the capillary Hamiltonian, i.e., Eq. (11) (up to the quartic term), is demonstrated by the discrepancy between the width of the interface as calculated directly from our simulation,

$$
w_{s}^{2}=\left\langle(h(\mathbf{x})-\langle h(\mathbf{x})\rangle)^{2}\right\rangle,
$$

and that as calculated from Eq. (11),

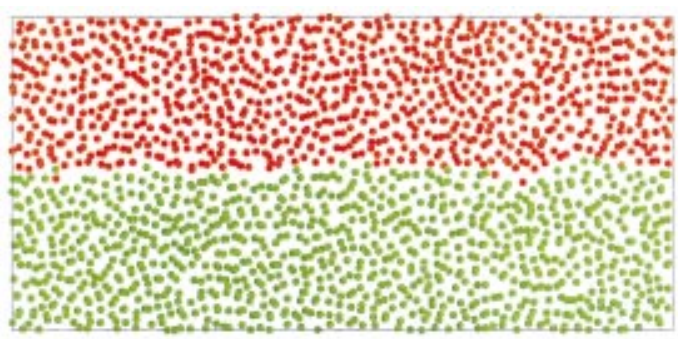

(a)
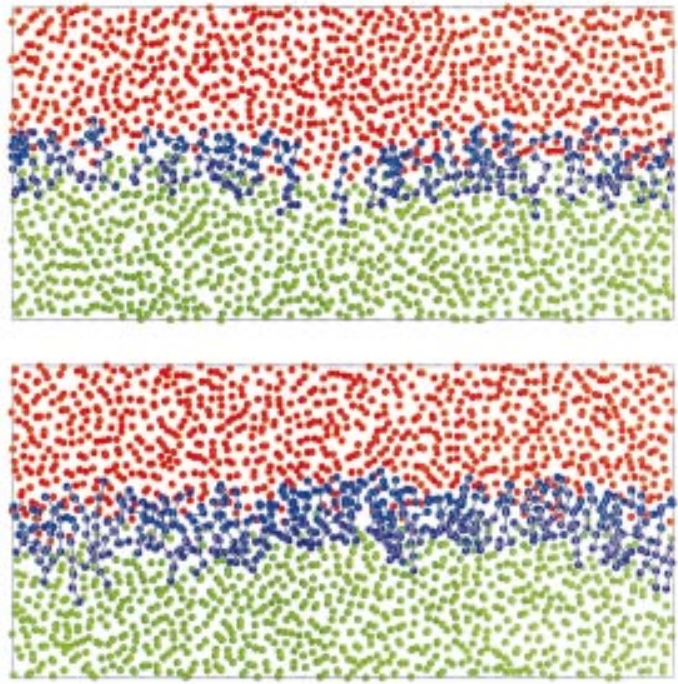

$(c)$

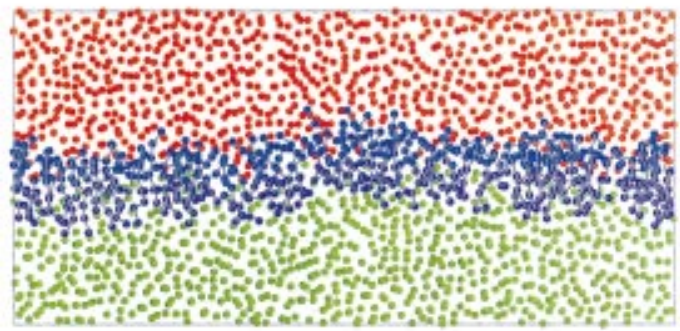

$(d)$

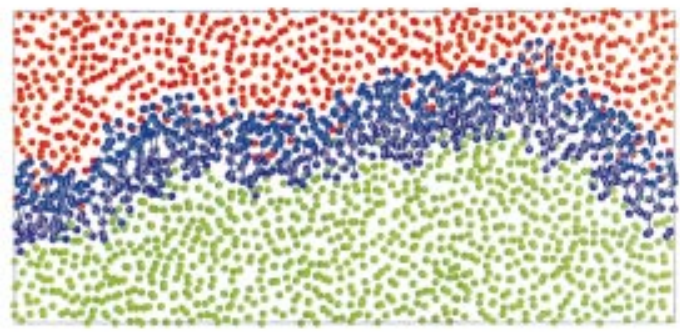

(e)

FIG. 2. (Color) The same as Fig. 1, but for surfactants with length $l_{s}=8$. Interface coverages shown correspond to (a) $N_{s}=0$, (b) $N_{s}=500$, (c) $N_{s}$ $=750$, (d) $N_{s}=1000$, and (e) $N_{s}=1250$.

$$
w_{c}^{2}=\frac{k_{\mathrm{B}} T}{4 \pi \sigma}\left[\ln \left(\frac{q_{\max }^{2}}{q_{\min }^{2}}\right)+\ln \left(\frac{\kappa+\frac{\sigma}{q_{\min }^{2}}}{\kappa+\frac{\sigma}{q_{\max }^{2}}}\right)\right],
$$

where $q_{\min }=\pi / L_{x y}$ and $q_{\max }=2 \pi / a$ and $a$ is the size of a plaquette, and using the values of $\sigma$ and $\kappa$ obtained from the simulation (see below). In Fig. 7, the width calculated di- 

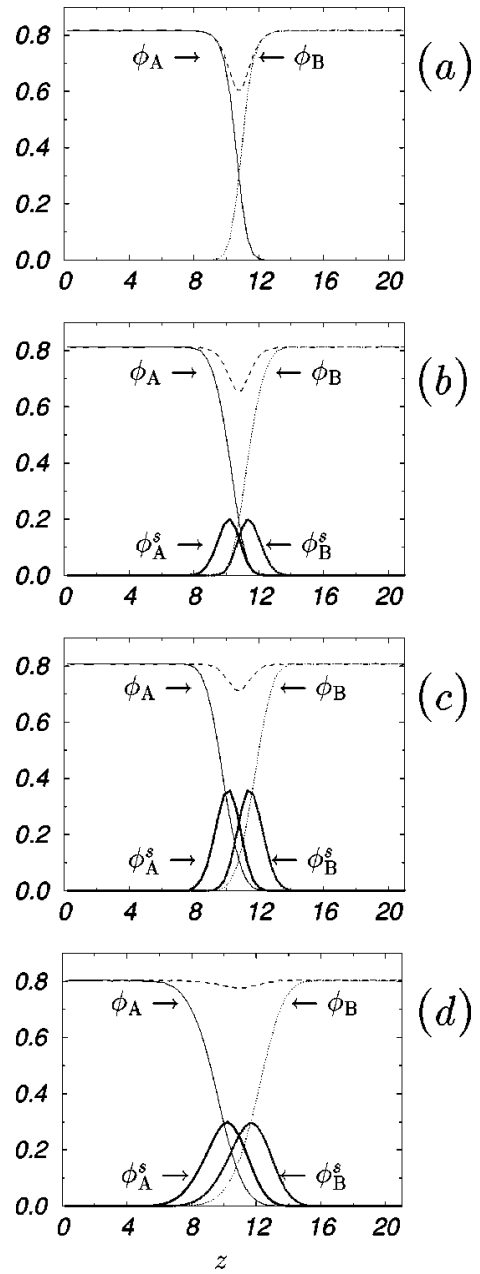

FIG. 3. Number density profiles (in unit of $\delta^{-3}$ ) along the $z$-axis for the ternary systems containing surfactants of length $l_{s}=4$ with interface coverages (a) $N_{s}=0$, (b) $N_{s}=500$, (c) $N_{s}=1000$, and (d) $N_{s}=1400$. The dashed lines correspond to the overall fluid number density.

rectly from the simulation, $w_{s}$, is plotted together with $w_{c}$, for the systems with $l_{s}=4$. Clearly the capillary Hamiltonian underestimate the interfacial width.

The interfacial tension and bending rigidity for the two surfactant lengths, $l_{s}=4$ and $l_{s}=8$, are shown in Figs. 8(a) and $8(b)$, respectively, for many surfactant interface coverages. For both surfactant lengths, the interfacial tension decreases monotonically with increasing the surfactant interface coverage and/or the surfactant chain length. The decrease in the interfacial tension becomes stronger as the surfactant chains become longer. The reduction in the interfacial tension is due to the crowding of the surfactants at the interface which induce a negative surface pressure. For the surfactants with length $l_{s}=4$, we find from extrapolation that the interfacial tension vanishes at roughly $N_{s}=1450$. Indeed, when we performed a simulation for $N_{s}=1500$, we found that the interfacial width reached values around the system size in the $z$-direction, implying that the interface becomes unstable at this surfactant interface coverage.

Figure 8(b) shows that for both chain lengths, the bending modulus changes nonmonotonically with the surfactant interface coverage. Indeed, for small surfactant interface coverages, the bending modulus decreases with increasing
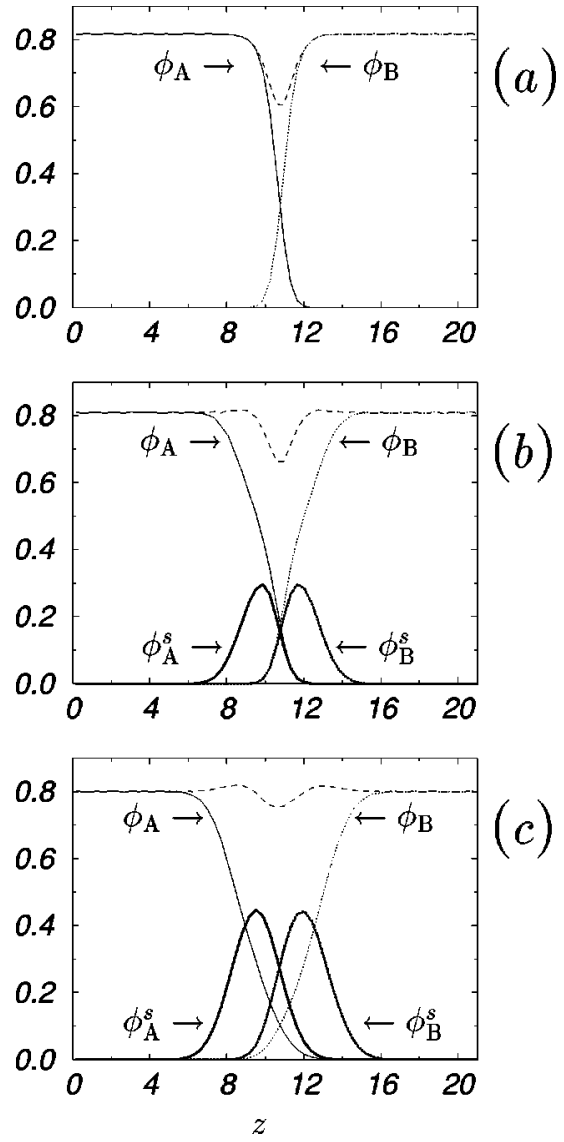

FIG. 4. The same as Fig. 3, but for the surfactants of length $l_{s}=8$. Figures shown correspond to (a) $N_{s}=0$, (b) $N_{s}=500$, and (c) $N_{s}=1000$.

surfactant interface coverage, but then it increases as the interface coverage is further increased. Moreover, the increase of the bending rigidity modulus with the surfactant interface coverage becomes steeper as the surfactants are made longer. Our results for the behavior of the bending rigidity in terms of the surfactant interfacial coverage are consistent with the recent calculations of Laradji and Desai ${ }^{18}$ for diblock copolymers at homopolymer-homopolymer interfaces. This nonmonotonic behavior of the bending rigidity can be related to the nature of the phase transition from the two-phase region to the disordered or lamellar phase. Indeed, for short (weak) surfactants, the transition from the two-phase region (corresponding to the water-rich phase in coexistence with the oilrich phase) to the disordered phase, as surfactant concentra-

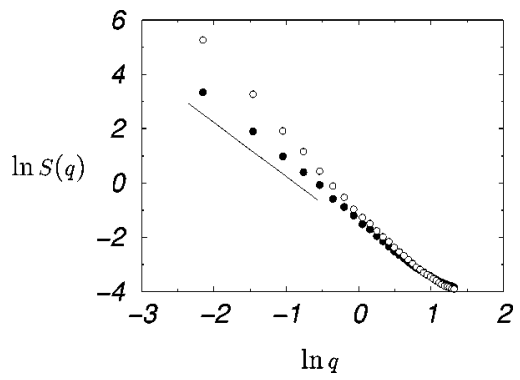

FIG. 5. $\ln S(q)$ vs $\ln q$ for the case without surfactants (-) and for the case of surfactants $(\bigcirc)$ of length $l_{s}=8$ and $N_{s}=1000$. The slope of the straight line is -2 . 


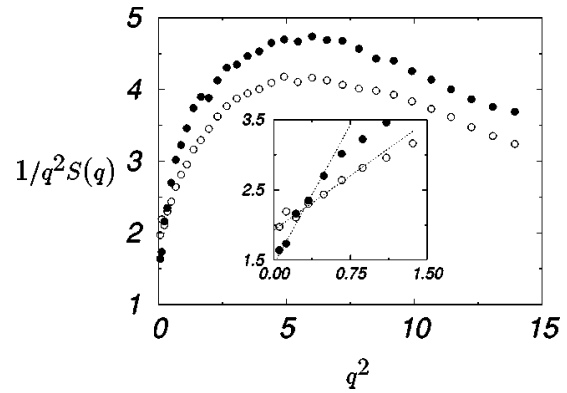

FIG. 6. $1 / q^{2} S(q)$ vs $q^{2}$ for the system with four monomers long surfactants and $N_{s}=750\left(-\right.$ ) and for the eight monomers long surfactants and $N_{s}$ $=750(\bigcirc)$.

tion is increased, is continuous. In this case, the excess interfacial energy must vanish at the transition, implying that both the interfacial tension and bending rigidity must vanish as well at the transition. Thus, the bending rigidity coefficient is expected to decrease with increasing surfactant concentration for short surfactants. As surfactant chains are made longer, the trenary mixture undergoes a discontinuous phase transition toward a microemulsion or a lamellar phase as surfactant concentration is increased. The internal interfaces between the oil-rich regions and water-rich regions in the microemulsion or lamellar phases are characterized by a significant value for the bending rigidity coefficient. Therefore, the bending rigidity is expected to increase with increasing surfactant concentration for long (strong) surfactants. For moderately long surfactants, a decrease followed by an increase of the bending rigidity coefficient is therefore conceivable. In order to explain more quantitatively this nonmonotonic behavior of the bending rigidity coefficient, we developed and investigated, in Appendix A, a GinzburgLandau model for an interface containing surfactants. The model is based on three local fields corresponding to the interfacial height, a surfactant concentration field and a surfactant orientation field. We believe that this model captures the most important properties of the system. In particular, the model accounts for the fact that a local splay in the surfactant monolayer induces a local spontaneous curvature which is proportional to the magnitude of the gradient in the orientation field. We found that a Gaussian treatment of the model leads to a decrease of the interfacial tension. On the other hand, the renormalization of the bending modulus is due to

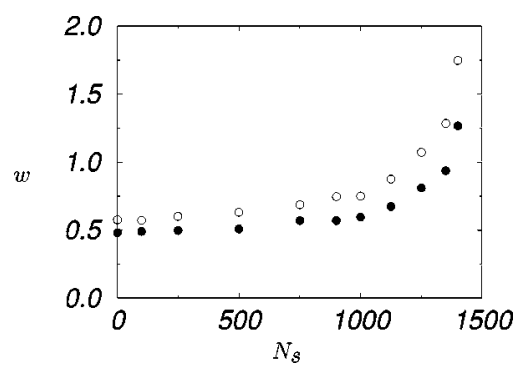

FIG. 7. The interfacial width as a function of surfactant amount for the short surfactants, $l_{s}=4$. The data with symbol $(\bigcirc)$ corresponds to the width obtained directly from the simulation using Eq. (15), and the data with the symbol, $\bullet$, correspond to the width as obtained from Eq. (16).
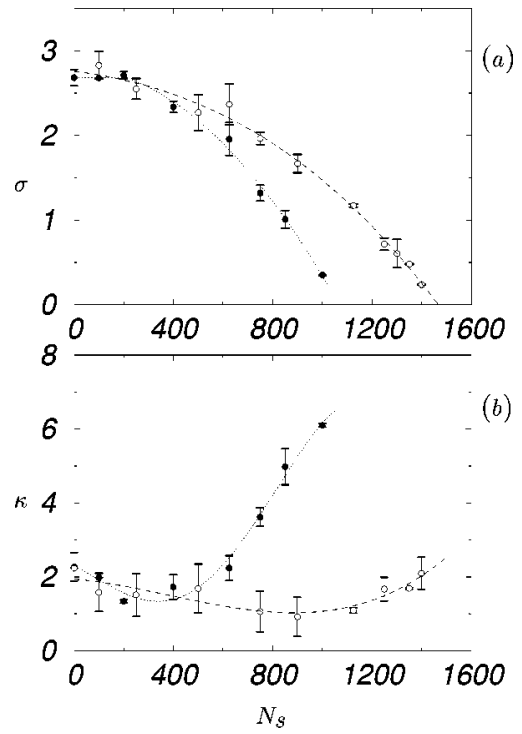

FIG. 8. The interfacial tension (a) and the bending rigidity modulus (b) vs the number of surfactants for surfactants of length $l_{s}=4(\bigcirc)$ and $l_{s}=8$ (๑). The solid and dotted lines are simply guides for the eye.

two competing effects: The preference of surfactants to align parallel to each other tend to increase the bending rigidity from its value for a bare surface; however, a local fluctuation in the orientation of surfactants lead to a decrease of the bending rigidity coefficient. Moreover, the later effect is more important for low surfactant concentrations. It is worthwhile noting that in our calculations, we found that the decrease in the bending rigidity is due to fluctuations in the orientation field of surfactants, i.e., in the conformation of surfactants, but not due to local concentration fluctuations. Indeed, very similar results were recently found by Matsen ${ }^{19}$ through a self-consistent mean field theory of diblock copolymers at homopolymer-homopolymer interfaces, a theory which accounts for conformation fluctuations of polymers, but not for composition fluctuations.

Finally, let us attempt to investigate the scaling of the interfacial tension and mean bending modulus with the surfactant interface coverage. In the wet brush regime, i.e., in the case when the polymers penetrate the homopolymer regions, scaling arguments ${ }^{17,28}$ predict that the excess in the interfacial tension behaves as

$$
\sigma_{0}-\sigma \propto c_{s}^{5 / 3} .
$$

The bending modulus scales as

$$
\kappa-\kappa_{0} \propto c_{s}^{7 / 3} \text {. }
$$

On the other hand, when the surfactants do not penetrate the $\mathrm{A}$ and $\mathrm{B}$ regions, i.e., in the case of a dry brush, scaling arguments ${ }^{14,19}$ predict that

$$
\sigma_{0}-\sigma \propto c_{s}^{3},
$$

and that the bending rigidity scales as

$$
\kappa-\kappa_{0} \propto c_{s}^{5} .
$$

In Fig. 9, the excess in the interfacial tension is shown in a double-logarithmic plot as a function of $c_{s}$. The agreement between our numerical results and Eq. (17) for both the short 


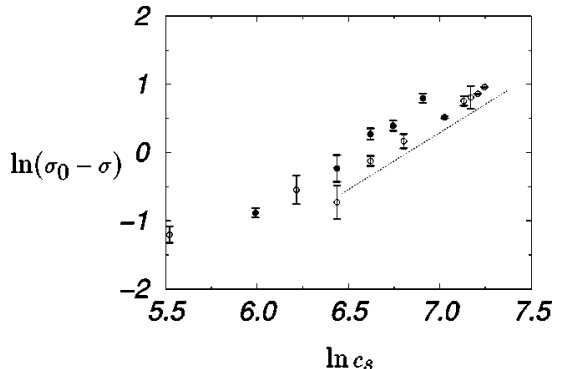

FIG. 9. $\ln \left(\sigma_{0}-\sigma\right)$ vs $\ln c_{s}$ for $l_{s}=4(\bullet)$ and $l_{s}=8(\bigcirc)$. The slope of the dotted line is $5 / 3$.

and long surfactants is indeed very good. For the bending modulus, an analysis of the data for $l_{s}=8$ on a double logarithmic scale was not possible due to the lack of points with $\kappa$ larger than that for a bare interface. However, a direct fit of the data actually leads to an exponent which is consistent with that for wet brushes.

\section{CONCLUSIONS}

In summary, we have in this paper presented an approach for deriving the leading elastic coefficients of a surfactant monolayer from molecular dynamics simulations through the analysis of the interfacial capillary fluctuations. We considered a ternary mixture of two simple fluids, A and $\mathrm{B}$, and flexible surfactants, each corresponding to a strand of A-monomers attached to an equally long strand of B-monomers. A wide range of surfactant interface coverages and two surfactant chain lengths were considered. The analysis of the structure factor of the capillary modes showed that higher order terms in the effective interfacial Hamiltonian are always important. Our main results can be summarized as follows: The interfacial tension decreases with increasing interface coverage of surfactants and/or the surfactant chain length. On the other hand, the effect of surfactants on the bending modulus, $\kappa$, is nonmonotonic. Namely, $\kappa$ decreases with increasing surfactant interface coverage, $c_{s}$, for small values of $c_{s}$, but then $\kappa$ increases with further increase of $c_{s}$. The increase of $\kappa$ with $c_{s}$ becomes steeper as the surfactant chain length is increased.

We also found that the minimum of $\kappa$ occurs at smaller values of $c_{s}$ as the surfactant chain length is increased. These results which confirm very nicely previous calculations of Laradji and Desai ${ }^{17}$ based on a self-consistent Gaussian theory of anisotropic fluctuations for diblock copolymers at homopolymer-homopolymer interfaces, are also verified by a Ginzburg-Landau model for a fluid-fluid interface containing surfactants. The other important elastic coefficient, corresponding to the saddle splay modulus, could not be inferred from our calculations due to the fact the interfaces in our simulations are planar and that the contribution of the saddle-splay term to the interfacial Hamiltonian is a topological constant.

\section{ACKNOWLEDGMENTS}

The authors would like to thank Professor Søren Toxvaerd and Dr. Paz Padilla for useful discussions, and Dr.
Bernd Dammann for his participation in the early stages of this project. This work was supported by the Natural Sciences and Engineering Research Council of Canada, the Danish Natural Science Research Council and the Danish Technical Research Council. O.G.M. is an associate fellow of the Canadian Institute for Advanced Research.

\section{APPENDIX: A FIELD-THEORETICAL MODEL FOR AN INTERFACE CONTAINING SURFACTANTS}

In this appendix, we will propose and study a simple interfacial model with surfactants. The free energy functional of the model which is derived from Ginzburg-Landau models for ternary mixtures of water-oil-surfactant ${ }^{29,30}$ is given by

$$
\begin{aligned}
\mathcal{F}(\{h\},\{\phi\},\{\mathbf{S}\})= & \int^{(\mathcal{A})} d \mathbf{x}\left[\sigma \sqrt{1+(\boldsymbol{\nabla} h)^{2}}+\frac{\kappa}{2}(\boldsymbol{\nabla} \cdot \hat{\mathbf{n}})^{2}\right. \\
& +\frac{a}{2} \phi^{2}+\frac{c}{2}(\boldsymbol{\nabla} \phi)^{2}-\mu_{s} \phi+\frac{g(\phi)}{2}|\mathbf{S}|^{2} \\
& -f(\phi) \hat{\mathbf{n}} \cdot \mathbf{S}+\frac{k(\phi)}{2}(\boldsymbol{\nabla} \cdot \mathbf{S})^{2} \\
& \left.-\frac{l(\phi)}{2}(\boldsymbol{\nabla} \cdot \hat{\mathbf{n}})(\boldsymbol{\nabla} \cdot \mathbf{S})\right],
\end{aligned}
$$

where $\mathcal{A}$ is the projected area of the interface. $\phi$ is the surfactant interfacial concentration field; $\mathbf{S}$ is a vector field representing the local surfactant orientation at the interface. $a, c$, and the surfactant chemical potential $\mu_{s}$ are positive constants, and the functions of $\phi, f, g, k$ and $l$ assume positive values. The two-dimensional gradient is $\boldsymbol{\nabla}=\partial_{x} \hat{\mathbf{x}}+\partial_{y} \hat{\mathbf{y}}$, and $\hat{\mathbf{n}}$ is a unit vector normal to the interface and is given by

$$
\hat{\mathbf{n}}=-\frac{1}{\sqrt{1+(\boldsymbol{\nabla} h)^{2}}}\left(\partial_{x} h \hat{\mathbf{x}}+\partial_{y} h \hat{\mathbf{y}}-\hat{\mathbf{z}}\right) .
$$

The term $(\nabla \phi)^{2}$ indicates that surfactants tend to homogenously distribute throughout the interface. The term $-f(\phi) \hat{\mathbf{n}} \cdot \mathbf{S}$ mimics the fact that surfactants prefer to orient themselves normal to the interface. The term $[g(\phi) / 2]|\mathbf{S}|^{2}$ keeps the orientation field from diverging and is therefore essential. Finally the last term is simply the usual bending term $(\kappa / 2)\left(\nabla^{2} h\right)^{2}$, but in the presence of surfactants it states that the interface prefers to curve with a local spontaneous curvature arising from the local arrangement of surfactants. The sum of the three terms, $\kappa(\boldsymbol{\nabla} \cdot \hat{\mathbf{n}})^{2}-l(\phi)(\boldsymbol{\nabla} \cdot \hat{\mathbf{n}})(\boldsymbol{\nabla} \cdot \mathbf{S})$ $+k(\phi)(\boldsymbol{\nabla} \cdot \mathbf{S})^{2}$ can be rewritten as

$$
\frac{\kappa}{2}\left(\boldsymbol{\nabla} \cdot \hat{\mathbf{n}}-\frac{l(\phi)}{2 \kappa} \boldsymbol{\nabla} \cdot \mathbf{S}\right)^{2}+\left(\frac{k(\phi)}{2}-\frac{l(\phi)^{2}}{8 \kappa}\right)(\boldsymbol{\nabla} \cdot \mathbf{S})^{2},
$$

which implies that depending on the local arrangement of surfactants, they induce a local spontaneous curvature, $l(\phi)(\boldsymbol{\nabla} \cdot \mathbf{S}) / 2 \kappa$, whose value decreases as the bare bending rigidity of the interface increases. From Eq. (A3), we find that in order to prevent the model from exhibiting unphysical short-wavelength instabilities the coefficient of $(\boldsymbol{\nabla} \cdot \mathbf{S})^{2}$ must remain positive. This leads to the condition 


$$
l(\phi) \leqslant 2 \sqrt{\kappa k(\phi)} .
$$

The mean-field solution of the model is obtained from minimizing the free-energy functional with respect to the three fields, leading to

$$
\begin{aligned}
& h^{(0)}(\mathbf{x})=\text { const, } \\
& \mathbf{S}^{(0)}=\frac{f\left(\phi^{(0)}\right)}{g\left(\phi^{(0)}\right)} \hat{\mathbf{z}},
\end{aligned}
$$

and $\phi^{(0)}$ being solution of

$$
a g^{2}(\phi) \phi-f^{\prime}(\phi) f(\phi) g(\phi)+\frac{g^{\prime}(\phi)}{2} f^{2}(\phi)=\mu_{s} g(\phi) \text {. }
$$

We now expand $h, \phi$ and $\mathbf{S}$ around their mean-field values:

$$
\begin{aligned}
& \phi(\mathbf{x})=\phi^{(0)}+\varphi(\mathbf{x}), \\
& \mathbf{S}(\mathbf{x})=\mathbf{S}^{(0)}+\mathbf{S}(\mathbf{x})=\mathbf{S}^{(0)}+s_{x}(\mathbf{x}) \hat{\mathbf{x}}+s_{y}(\mathbf{x}) \hat{\mathbf{y}}+s_{z}(\mathbf{x}) \hat{\mathbf{z}} .
\end{aligned}
$$

(If $h^{(0)}$ is zero, then $h$ itself is a fluctuation). The free-energy functional of the system can therefore be expanded in terms of the fluctuating fields and can be written as

$$
\mathcal{F}=\mathcal{F}^{(0)}+\mathcal{F}^{(1)}+\mathcal{F}^{(2)}+\cdots,
$$

where $\mathcal{F}^{(0)}$ is the mean-field free energy. $\mathcal{F}^{(1)}$ vanishes since the mean-field solution minimizes the free energy functional, Eq. (A1), and the quadratic term $\mathcal{F}^{(2)}$ is given by

$$
\begin{aligned}
\mathcal{F}^{(2)}(\{h\},\{\varphi\},\{\mathbf{s}\})= & \int d \mathbf{x}\left[\frac{\sigma}{2}(\boldsymbol{\nabla} h)^{2}+\frac{\kappa}{2}\left(\nabla^{2} h\right)^{2}+\left(\frac{a}{2}-\frac{f_{0}^{\prime \prime}}{2} s_{z}^{(0)}+\frac{g_{0}^{\prime \prime}}{4} s_{z}^{(0) 2}\right) \varphi^{2}+\frac{c}{2}(\boldsymbol{\nabla} \varphi)^{2}+\left(g_{0}^{\prime} s_{z}^{(0)}-f_{0}^{\prime}\right) \varphi s_{z}+\frac{g_{0}}{2}|\mathbf{s}|^{2}\right. \\
& \left.\left.+\frac{k_{0}}{2}\left(\left(\partial_{x} s_{x}\right)^{2}+\left(\partial_{y} s_{y}\right)^{2}+2 \partial_{x} s_{x} \partial_{y} s_{y}\right)\right)+f_{0}\left(s_{x} \partial_{x} h+s_{y} \partial_{y} h\right)+\frac{l_{0}}{2}\left(\nabla^{2} h \partial_{x} s_{x}+\nabla^{2} h \partial_{y} s_{y}\right)\right]
\end{aligned}
$$

where $f_{0}=f\left(\phi^{(0)}\right)$, and $f_{0}^{\prime}$ and $f_{0}^{\prime \prime}$ are first and second derivatives of $f$ at $\phi^{(0)}$ (similar notations are used for $g, k$ and $l$ ). In the free energy above, Gaussian fluctuations in the surfactant concentrations are decoupled from the interfacial fluctuations. Therefore, renormalization of the interfacial tension, $\sigma$, and the bending coefficient, $\kappa$, at the Gaussian level, will result from coupling between fluctuations in the $x$ - and $y$-components of the orientational field on one hand and the capillary field, $h$, on the other hand. Consequently, only terms depending on $s_{x}, s_{y}$ and $h$ are relevant for us. After rewriting the relevant part of the quadratic free energy functional in terms of the Fourier components of $h_{\mathbf{q}}, s_{x, \mathbf{q}}$ and $s_{y, \mathbf{q}}$, and after Gaussian integration of the orientational fields, we find the following effective free-energy functional in terms of $h_{\mathbf{q}}$ :

$$
\mathcal{F}_{\mathrm{eff}}^{(2)}(\{h\})=\int d \mathbf{q} S^{-1}(q) h_{\mathbf{q}} h_{-\mathbf{q}},
$$

where the inverse of the structor factor is given by

$$
S^{-1}(q)=\frac{1}{2 k_{B} T}\left[\sigma q^{2}+\kappa q^{4}-\frac{\left(f_{0}+\frac{l_{0}}{2} q^{2}\right)^{2} q^{2}}{g_{0}+k_{0} q^{2}}\right],
$$

which can be expanded for small wavevectors as

$$
S^{-1}(q)=\frac{1}{2 k_{\mathrm{B}} T}\left[\tilde{\sigma} q^{2}+\tilde{\kappa} q^{4}+\mathcal{O}\left(q^{6}\right)\right]
$$

where the renormalized interfacial tension and bending rigidity are given by

$$
\tilde{\sigma}=\sigma-\frac{f_{0}^{2}}{g_{0}}
$$

$$
\tilde{\kappa}=\kappa-\frac{l_{0} f_{0}}{g_{0}}+\frac{k_{0} f_{0}^{2}}{g_{0}^{2}} .
$$

Since $f_{0}$ and $g_{0}$ are positive, the surfactants lead to an increase of the interfacial tension, as expected. On the other hand, we will see that surfactants can lead to either an increase or a decrease of the bending rigidity, depending on the value of $\phi^{(0)}$. It is worthwhile making the remark that the structure factor, in Eq. (A10), is always positive as long as the interfacial tension $\tilde{\sigma}>0$ and that the stability condition, Eq. (A4), is built-in to the model.

We now discuss the behavior of $\tilde{\kappa}-\kappa$ as a function of $\psi=\phi^{(0)}$ and surfactant chain length $l_{s}$. First, as the surfactant concentration approaches zero, the additional part to the Hamiltonian, due to surfactants, should approach zero as well. The effect of surfactant should also diminish as the surfactant chain length decreases. The functions $f, g, k$; and $l$ should therefore approach zero. Let us assume that this approach is algebraic. For small $\phi^{(0)}$, we can then write

$$
k_{0} \sim \psi^{\alpha} l_{s}^{\alpha^{\prime}}, \text { where } \alpha, \alpha^{\prime}>0
$$

Moreover, the orientation order parameter, $S_{z}^{(0)}$ should also approach zero as $\phi^{(0)}$ decreases. Obviously, $S_{z}^{(0)}$ should also decrease with decreasing surfactant length, $l_{s}$. From Eq. (A6), we can therefore write

$$
\frac{f_{0}}{g_{0}} \sim \psi^{\beta} l_{s}^{\beta^{\prime}}, \text { where } \beta, \beta^{\prime}>0 .
$$

In order to satisfy the stability condition, Eq. (A4), we can also write

$$
l_{0} \sim \psi^{\gamma} l_{s}^{\gamma^{\prime}}, \gamma>\frac{\alpha}{2} ; \gamma^{\prime}>\frac{\alpha^{\prime}}{2} .
$$


Therefore, for small $\psi$, the bending rigidity excess can be written as

$$
(\tilde{\kappa}-\kappa) \sim \psi^{\alpha+2 \beta} l_{s}^{\alpha+2 \beta}-C \psi^{\gamma+\beta} l_{s}^{\gamma^{\prime}+\beta^{\prime}},
$$

which exhibits a minimum at a certain value of $\psi_{c}$ if $\alpha / 2$ $<\gamma<\alpha+\beta$. Moreover, the concentration $\psi_{c}$ decreases as the chain length is increased. These results agree well with the self-consistent field theory of anisotropic fluctuations recently developed by Laradji and Desai ${ }^{17}$ for diblock copolymers at homopolymer-homopolymer interfaces.

${ }^{1}$ G. Gompper and M. Schick, in Self Assembling Amphiphilic Systems (Academic, New York, 1994).

${ }^{2}$ M. Laradji, H. Guo, M. Grant, and M. J. Zuckermann, in Advances in Chemical Physics, edited by I. Prigogine and S. A. Rice (Wiley, New York, 1995), Vol. LXXXIX, p. 159.

${ }^{3}$ W. Helfrich, Z. Naturforsch. A 33, 305 (1981).

${ }^{4}$ J. S. Huang, S. T. Milner, B. Farago, and D. Richter, Phys. Rev. Lett. 59, 2600 (1987).

${ }^{5}$ B. Farago, D. Richter, J. S. Huang, S. A. Safran, and S. T. Milner, Phys. Rev. Lett. 65, 3348 (1990).

${ }^{6}$ T. Hellweg and D. Langevin, Phys. Rev. E 57, 6825 (1998).

${ }^{7}$ G. Gompper and S. Zschocke, Europhys. Lett. 16, 731 (1991).

${ }^{8}$ M. M. Telo da Gama and K. E. Gubbins, Mol. Phys. 59, 227 (1986); M. M. Telo da Gama, ibid. 62, 585 (1987).

${ }^{9}$ I. Szleifer, D. Kramer, A. Ben-Shaul, D. Roux, and W. M. Gelbert, Phys. Rev. Lett. 60, 1966 (1988); I. Szleifer, D. Kramer, A. Ben-Shaul, W. M. Gelbart, and S. A. Safran, J. Chem. Phys. 92, 6800 (1990).
${ }^{10}$ R. S. Cantor and P. M. McIlroy, J. Chem. Phys. 90, 4431 (1989); 99, 7124 (1993); 104, 8082 (1996).

${ }^{11}$ B. Smit, P. A. J. Hilbers, K. Esselink, L. A. M. Rupert, N. M. Overns, and A. G. Schlijper, Nature (London) 348, 624 (1990); J. Phys. Chem. 95, 6361 (1990).

${ }^{12}$ R. Goetz, G. Gompper, and R. Lipowsky, Phys. Rev. Lett. 82, 221 (1999).

${ }^{13}$ J. Noolandi and K. M. Hong, Macromolecules 15, 727 (1981); 14, 736 (1981); K. M. Hong and J. Noolandi, ibid. 15, 482 (1982); 17, 1531 (1984).

${ }^{14}$ Z.-G. Wang and S. A. Safran, J. Phys. (France) 51, 185 (1990); J. Chem. Phys. 94, 679 (1991).

${ }^{15}$ M. W. Matsen and M. Schick, Macromolecules 26, 3878 (1993).

${ }^{16}$ L. Leibler, Macromolecules 13, 1602 (1982).

${ }^{17}$ M. Laradji and R. C. Desai, J. Chem. Phys. 108, 4662 (1998).

${ }^{18}$ M. Laradji, A.-C. Shi, R. C. Desai, and J. Noolandi, Phys. Rev. Lett. 78, 2577 (1997); M. Laradji, A.-C. Shi, J. Noolandi, and R. C. Desai, Macromolecules 30, 3242 (1997).

${ }^{19}$ M. Matsen, J. Chem. Phys. 110, 4658 (1999).

${ }^{20}$ M. Müller and M. Schick, J. Chem. Phys. 105, 8885 (1996).

${ }^{21}$ A. Werner, F. Schmid, and M. Müller, J. Chem. Phys. 110, 5370 (1999).

${ }^{22}$ J. D. Weeks, D. Chandler, and H. C. Andersen, J. Chem. Phys. 54, 5237 (1971).

${ }^{23}$ S. Toxvaerd and E. Velasco, Mol. Phys. 86, 845 (1995).

${ }^{24}$ S. Nosé, Mol. Phys. 52, 255 (1984).

${ }^{25}$ W. G. Hoover, Phys. Rev. A 31, 255 (1985).

${ }^{26}$ S. Toxvaerd, Mol. Phys. 31, 1695 (1991).

${ }^{27}$ S. H. Kim and W. H. Jo, J. Chem. Phys. 110, 12193 (1999).

${ }^{28}$ L. Leibler, Makromol. Chem., Macromol. Symp. 16, 1 (1988).

${ }^{29}$ K. Chen, C. Jayaprakash, R. Pandit, and W. Wenzel, Phys. Rev. Lett. 65, 2736 (1990)

${ }^{30}$ J. Melenkevitz and S. H. Javadpour, J. Chem. Phys. 107, 623 (1997). 